\title{
Diseño y análisis energético de un sistema de refrigeración autónomo para el traslado de vacunas
}

\section{Design and energy analysis of an autonomous cooling system for the transportation of vaccines}

\author{
VALLE-HERNÁNDEZ, Julio†*, LOZANO-OLMEDO, Britania Junery, DÍAZ-MONROY, Joanna y \\ ROMÁN-AGUILAR, Raúl
}

Universidad Autónoma del Estado de Hidalgo. Escuela Superior de Apan, Ingeniería en Tecnología del Frío

ID $1^{\text {er }}$ Autor: Julio, Valle-Hernández / ORC ID: 0000-0001-8957-0066, Researcher ID Thomson: 0-7339-2018, CVU CONACYT ID: 210743

ID $1^{\text {er }}$ Coautor: Britania Junery, Lozano-Olmedo / ORC ID: 0000-0002-2720-2978

ID $2^{\text {do }}$ Coautor: Joanna, Díaz-Monroy / ORC ID: 0000-0002-7808-4609

ID $3^{\text {er }}$ Coautor: Raúl, Román-Aguilar / ORC ID: 0000-0003-0753-2352, Researcher ID Thomson: 1-6746-2018, CVU CONACYT ID: 165332

DOI: $10.35429 /$ JEA.2019.21.6.14.23

Recibido: 19 de Agosto, 2019; Aceptado Noviembre, 30, 2019

\section{Resumen}

La vacunación es uno de los métodos más importantes de promoción de la salud en el mundo, para preservar la eficacia de estos productos farmacológicos el rango de temperaturas de manejo es de entre $2^{\circ} \mathrm{C}$ y $8^{\circ} \mathrm{C}$. Los enfriadores termoeléctricos son dispositivos de estado sólido que convierten la energía eléctrica en energía térmica, aunque están destinados a pequeñas capacidades de enfriamiento que van del orden de 2 a 250 watts, son óptimos para el enfriamiento de sistemas portátiles. El presente trabajo describe el diseño y análisis energético de un sistema portátil para el traslado de vacunas en zonas rurales, el cual será alimentado con energía solar. El diseño integra la elección del aislamiento térmico, la geometría y funcionamiento del sistema. El análisis energético permite obtener la energía necesaria para mantener las vacunas a una temperatura entre $4^{\circ} \mathrm{C} \mathrm{y} 6^{\circ} \mathrm{C}$, a partir de la estimación de las cargas térmicas, y con ello la elección del sistema fotovoltaico más adecuado para garantizar el tiempo de autonomía necesario.

Sistema de refrigeración portátil, Traslado de vacunas, Autonomía energética

\begin{abstract}
Vaccination is one of the most important methods of promoting health in the world, to preserve the effectiveness of these pharmacological products the operation temperature range in between $2^{\circ} \mathrm{C}$ and $8^{\circ} \mathrm{C}$. Thermoelectric coolers are solid state devices that convert electrical energy into thermal energy, although they are destined to small capacities of cooling which are of the order of 2 to 250 watts, they are optimal for the cooling of portable systems. This paper describes the design and energy analysis of a portable system for the transfer of vaccines in rural areas, which will be powered by solar energy. The design integrates the choice of thermal insulation, geometry and operation of the system. Energy analysis allows to obtain the necessary energy to maintain the vaccines at temperatures between $4^{\circ} \mathrm{C}$ and $6^{\circ} \mathrm{C}$, from the estimation of the thermal loads, and thus the election of the photovoltaic system best suited to ensure the necessary autonomy time.
\end{abstract}

Portable cooling system, Transfer of vaccines, Energy autonomy

Citación: VALLE-HERNÁNDEZ, Julio, LOZANO-OLMEDO, Britania Junery, DÍAZ-MONROY, Joanna y ROMÁNAGUILAR, Raúl. Diseño y análisis energético de un sistema de refrigeración autónomo para el traslado de vacunas. Revista de Aplicaciones de la Ingeniería. 2019. 6-21: 14-23

\footnotetext{
* Correspondencia del Autor (Correo electrónico: julio_valle@ uaeh.edu.mx)

$\dagger$ Investigador contribuyendo como primer autor.
} 


\section{Introducción}

Según la OMS (2019), las vacunas son productos biológicos que generan inmunidad contra una enfermedad estimulando la producción de anticuerpos, ayudan a reforzar el sistema inmune, previniendo enfermedades y mejorando la calidad de vida de las personas. Para que una vacuna pueda proteger a una persona es necesario que se conserve entre $2{ }^{\circ} \mathrm{C}$ y $8{ }^{\circ} \mathrm{C}$ (ABB, 2009), desde la salida de los laboratorios hasta su aplicación, sin romper la cadena de frio, para así evitar que se reduzca la efectividad de la misma y poner en peligro a los pacientes que se les aplique.

Las personas que viven en zonas rurales son propensas a contraer enfermedades por no recibir atención médica preventiva, debido a que se encuentran en un lugar alejado de los centros de atención médica. Una de las enfermedades más importantes a combatir es la Influenza Estacional, donde la vacunación adecuada es de suma importancia para así evitar el contagio. Esta vacuna debe transportarse en contenedores refrigerados que la almacenen a la temperatura adecuada y la conserven durante toda la jordana en zonas rurales o de difícil suministro de energía.

Por otra parte, la refrigeración termoeléctrica está basada en el efecto Peltier, y constituye un sistema de conversión directa de electricidad en frío, sin pasar por energía mecánica ni térmica como ocurre con los sistemas convencionales de compresión o absorción (Winder, Ellis \& Lisensky, s.f.). Al tratarse de un sistema completamente estático, sin partes móviles, y alimentado únicamente por corriente continua, es muy útil en todas aquellas aplicaciones en donde la carga a refrigerar esté en movimiento (Villarrubia, 2001), como es el caso de las campañas de vacunación.

En el presente trabajo se propone el diseño y análisis energético de un contenedor para el transporte refrigerado de vacunas, contra la Influenza Estacional, mediante termoeléctricos alimentados con energía solar. El diseño se realizó a partir del cálculo de cargas térmicas, tomando en cuenta el número de vacunas dentro del sistema, el aislante térmico y la infiltración de calor desde el exterior.
El sistema de refrigeración termoeléctrico es alimentado por medio de un sistema fotovoltaico autónomo, el cual realiza tres funciones; la primera es transformar directamente la energía solar en energía eléctrica (Solar Fotovoltaica, 2002), la segunda función es almacenar adecuadamente la energía eléctrica generada y finalmente proveer la energía necesaria al sistema de refrigeración.

\section{Metodología}

La metodología para realizar el diseño y análisis energético del sistema de refrigeración consistió en seis pasos. En el primer paso se definió la ubicación geográfica de la zona rural en donde se pretendió hacer la distribución las vacunas, con la finalidad de determinar la radiación solar y la temperatura máxima a la que operó el sistema. El paso dos consistió en proponer el diseño del sistema de almacenamiento; geometría, medidas, materiales, etc. Posteriormente en el paso tres se realizó un análisis energético para calcular las cargas térmicas, tomando en cuenta el número de vacunas dentro del sistema, el aislante térmico, el tipo de material y las cargas térmicas por infiltración del exterior y por cambios de aire.

En el cuarto paso se eligió el termoeléctrico a partir de las condiciones requeridas por el sistema (número, tipo, capacidad y especificaciones). El paso cinco consistió en calcular la energía consumida por el dispositivo, partiendo de las características del sistema termoeléctrico elegido. Finalmente, en el paso seis se dimensionó el sistema fotovoltaico que provee la energía al sistema; configuración y dimensiones óptimas para cumplir con la temperatura requerida para almacenar las vacunas.

\section{Desarrollo}

\section{Ubicación geográfica y determinación de las características climatológicas}

En el presente trabajo se consideró la Zona del Valle del Mezquital que es una zona geográfica y cultural del estado de Hidalgo, la cual es habitada principalmente por el pueblo indígena otomí y esta se encuentra alejada de la zona urbana. En esta zona la temperatura máxima promedio que se presenta a lo largo del año es de $35^{\circ} \mathrm{C}$ y la irradiación solar mínima a lo largo del año es de $4.2 \mathrm{kWh} / \mathrm{m}^{2}$. 
En el gráfico 1 y en el gráfico 2 se observa la temperatura máxima promedio y la irradiación solar a lo largo de todo el año.

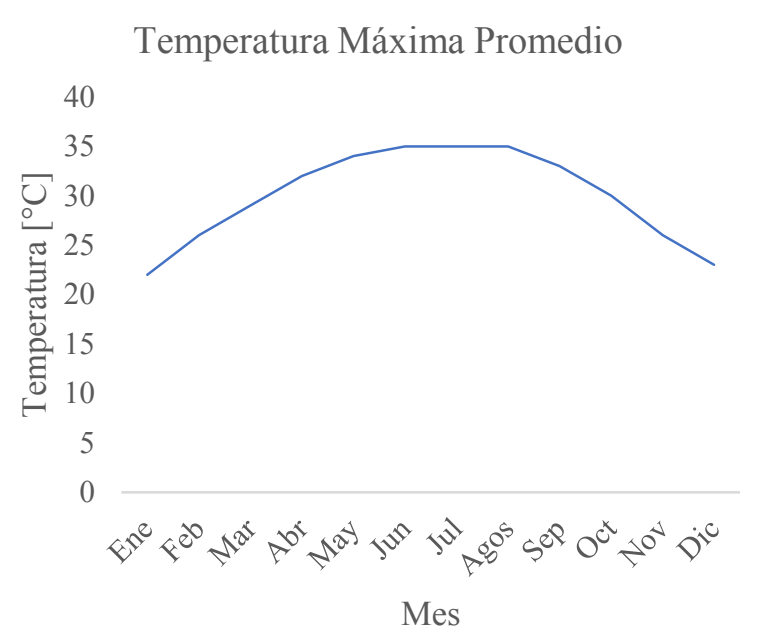

Gráfico 1 Temperatura máxima promedio anual Fuente: Diebel, 2019

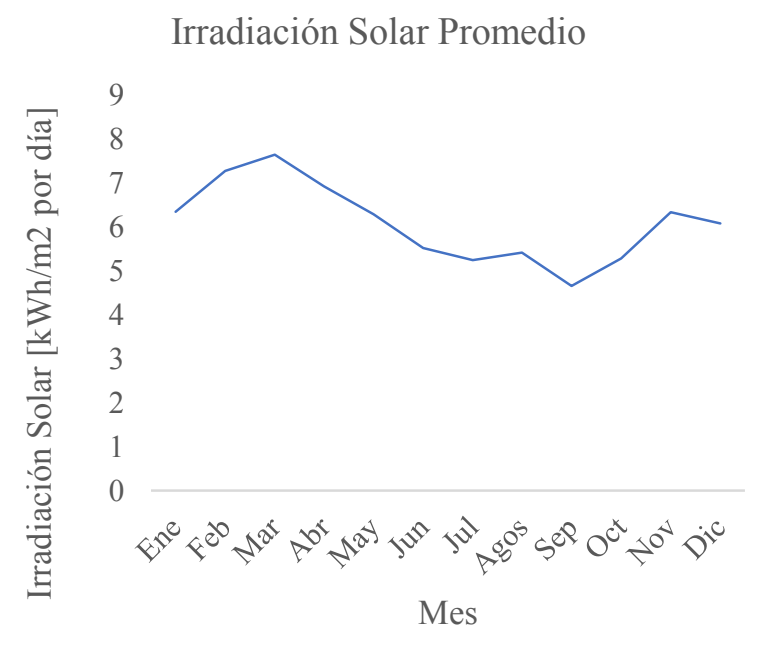

Gráfico 2 Irradiación solar promedio anual

Fuente: Nasa Power, 2019

\section{Diseño del equipo}

En el ámbito de la salud existen infinidad de equipos que tienen como propósito conservar la temperatura de vacunas. Su capacidad suele ser reducida debido a que utilizan recipientes con sustancias refrigerantes para mantener la temperatura interna a lo largo de la jornada. Para el diseño de este dispositivo no se tomó en cuenta el uso de los recipientes antes mencionados puesto que está en constante refrigeración por medio de celdas Peltier, evitar el uso de recipientes refrigerantes disminuye notablemente el peso total del dispositivo y aumenta su capacidad interna dejando más espacio para vacunas.
En el presente proyecto se plantea diseñar un dispositivo que garantice un sistema continuo de refrigeración, que optimice el espacio para vacunas y así tenga un fácil acceso a ellas sin aumentar su temperatura.

Una vez que las vacunas estén dentro de la cámara, estas no deben estar en contacto entre sí ni con las paredes. Esto permitirá que las vacunas se refrigeren correctamente además de que permite que la cámara no esté sobre cargada (Godoy, 2016).

En función del número y de las dimensiones del frasco que contiene a la vacuna, especificado en el subtema 3.2.1, se propuso un volumen y a partir de la capacidad de almacenamiento se determinó la altura y el radio que permitió minimizar el área de transferencia. Estas dimensiones se determinaron mediante las siguientes ecuaciones.

$$
r=\sqrt[3]{\frac{v}{2 \pi}}
$$

Donde:

$r=$ Radio $[\mathrm{m}]$

$v=$ Volumen $[\mathrm{L}]$

$h=\frac{v}{\pi r^{2}}$

Donde:

$h=$ Altura $[\mathrm{m}]$

$v=$ Volumen $[\mathrm{L}]$

$r=$ Radio $[\mathrm{m}]$

$A=2 \pi r(h+r)$

Donde:

$A=$ Área $\left[\mathrm{m}^{2}\right]$

$r=$ Radio $[\mathrm{m}]$

$h=$ Altura $[\mathrm{m}]$

En la siguiente tabla se muestran las dimensiones establecidas del dispositivo.

\begin{tabular}{|l|l|l|}
\hline Dimensión & \multicolumn{1}{c|}{ Valor } & Unidad \\
\hline Volumen & 3 & {$[\mathrm{~L}]$} \\
\hline Radio & 0.065 & {$[\mathrm{~m}]$} \\
\hline Altura & 0.226 & {$[\mathrm{~m}]$} \\
\hline Área & 0.12 & {$\left[\mathrm{~m}^{2}\right]$} \\
\hline
\end{tabular}

Tabla 1 Dimensiones del equipo

Fuente: Elaboración Propia

En la Figura 1 se puede observar el diseño propuesto del contenedor para transportar las vacunas durante las jornadas de vacunación. 


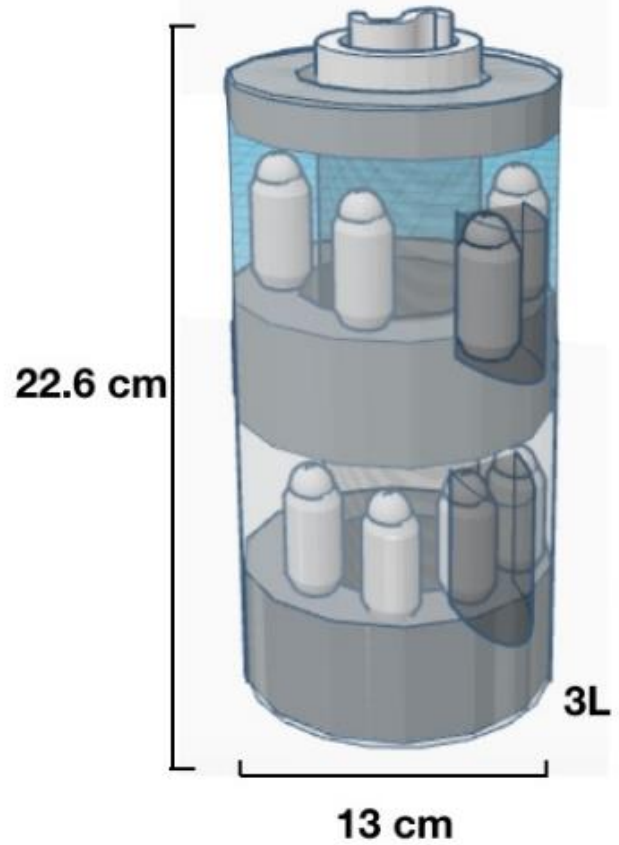

Figura 1 Diseño y dimensiones del contenedor de vacunas.

Fuente: Elaboración Propia

Material externo: polietileno de alta densidad resistente al impacto, liso, sólido, lavable, no corrosivo y no reactivo, con un coeficiente de conductividad térmica de 0.4 $\mathrm{W} / \mathrm{mK}$.

Tapa: sólida con sello hermético.

Se adiciono en la parte superior un ventilador de $7 \mathrm{~cm}$ de diámetro, una potencia de $5 \mathrm{~W}$ y un flujo volumétrico de $0.00523 \mathrm{~m}^{3} / \mathrm{s}$. Para asegurar que exista flujo de aire dentro del dispositivo.

\section{Capacidad del equipo de refrigeración}

Para Godoy (2016), un correcto dimensionamiento de un dispositivo de almacenamiento de vacunas, debe tomar en cuenta la cantidad de vacunas que se deberán contener en el sistema de refrigeración.

Este sistema de refrigeración debe estar en la capacidad de refrigerar al menos $100 \mathrm{ml}$ de vacunas que pueden estar contenidas en frascos de $5 \mathrm{ml}$ de capacidad, es decir, 20 frascos.

En la Figura 2 se pueden observar las medidas del frasco de la vacuna a refrigerar.

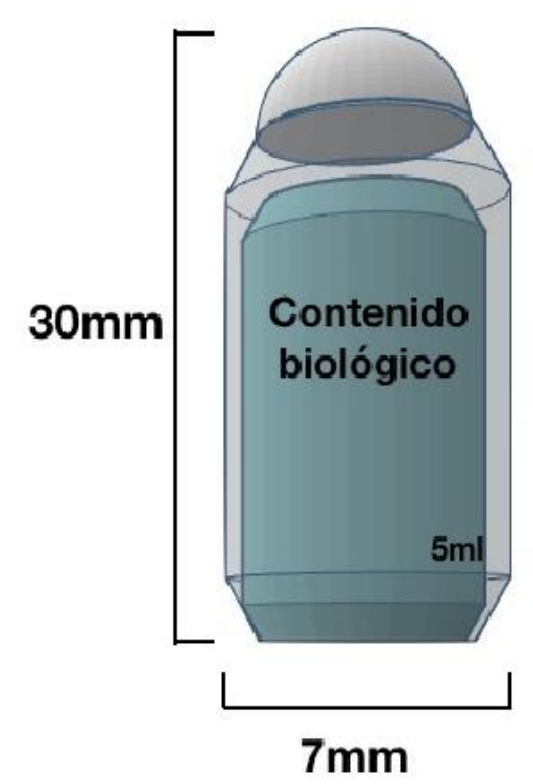

Figura 2 Dimensiones del frasco de una vacuna Fuente: Elaboración Propia

Para el caso de las vacunas contra la influenza AH1N1 cada dosis es de $0.5 \mathrm{ml}$, lo que se traduce en el suministro necesario para 200 personas en cada contenedor.

\section{Aislamiento térmico}

Uno de los principales inconvenientes a la hora de refrigerar o congelar productos biológicos son las pérdidas que se producen a través de las paredes. Una de las formas de reducir esta pérdida de calor, es seleccionar materiales con baja conducción térmica, que permita que la diferencia de temperaturas interna y externa sean máximas (Incropera, 1999).

Un material térmico tiene la característica de tener una baja conductividad térmica, además de ser resistente a las bajas temperaturas (BOHN, 2005). Para el transporte de producto biológico en la industria usualmente se recurre al aislamiento con poliuretano de alta densidad ("espuma de poliuretano") libre de CFC's, este material tiene un coeficiente de conductividad térmica de $0.038[\mathrm{~W} / \mathrm{mK}]$. Y según Thermos (2008) compañía certificada por UNICEF establece que el espesor para un aislante térmico en dispositivos de almacenamiento de vacunas es de $20 \mathrm{~mm}$. 


\section{Análisis energético}

\section{Cargas térmicas}

La carga térmica se define como la cantidad de calor que debe ser retirado del sitio a refrigerar para reducir o mantener la temperatura deseada. Para acondicionar un área la carga térmica se debe eliminar mediante el enfriamiento. La carga térmica total resulta de la suma de las cargas térmicas involucradas (HVACR, 2012). En el diseño propuesto únicamente se consideraron las cargas térmicas por transmisión a través de paredes, por producto y por infiltración por aire exterior. La temperatura del contenedor debe mantenerse entre 2 y $8^{\circ} \mathrm{C}$, considerando una temperatura máxima del ambiente en el mes de julio durante la segunda jornada de vacunación nacional de $35^{\circ} \mathrm{C}$.

\section{Carga térmica por producto}

Para calcular cuánto calor debe ser extraído del dispositivo se debe calcular la carga térmica que genera el producto de la siguiente manera:

$Q_{\text {prod }}=\left(\frac{m}{t} * C p * \Delta T\right)+\left(\frac{m_{v}}{t} * C p_{v} * \Delta T\right)$

Dónde:

$Q_{\text {prod }}=$ Calor extraído por producto $[W]$

$m=$ Masa del producto $[\mathrm{kg}]$

$m_{v}=$ Masa del envase de vidrio de la vacuna

[kg]

$t=$ Tiempo de traslado $[s]$

$C p=$ Calor específico arriba del punto de congelación $[\mathrm{J} / \mathrm{kgK}]$

$C p_{v}=$ Calor específico arriba del punto de congelación del vidrio [ $\mathrm{J} / \mathrm{kgK}]$

$\Delta T=$ Diferencia de temperatura $[K]$

En la Tabla 2, se pueden observar los parámetros utilizados para determinar la Carga Térmica por Producto.

\begin{tabular}{|l|r|l|}
\hline Parámetro & \multicolumn{1}{l}{ Valor } & Unidades \\
\hline$m$ & 0.1025 & $\mathrm{Kg}$ \\
\hline$m_{v}$ & 0.0885 & $\mathrm{Kg}$ \\
\hline$t$ & 28,800 & $\mathrm{~S}$ \\
\hline$C p$ & 4.18 & $\mathrm{~kJ} / \mathrm{kgK}$ \\
\hline$C p_{v}$ & 41.86 & $\mathrm{~J} / \mathrm{kgK}$ \\
\hline$\Delta T$ & 6 & $\mathrm{~K}$ \\
\hline
\end{tabular}

Tabla 2 Parámetros para obtener la carga térmica por producto de las vacunas

Fuente: Elaboración Propia

\section{Carga térmica generada por transmisión de paredes}

La carga térmica generada por infiltración a través del área del dispositivo se calculó mediante la siguiente ecuación:

$Q_{\text {paredes }}=A * U * \Delta T$

Donde:

$Q_{\text {paredes }}=$ Calor total de transmisión a través de las paredes $[W]$

$A=$ Área de transferencia de calor $\left[\mathrm{m}^{2}\right]$

$\Delta T=$ Diferencial de temperatura entre la temperatura interior y exterior $[K]$

$U=$ Coeficiente global de transferencia de calor $\left[W / m^{2} K\right]$, se calcula como:

$$
U=\frac{1}{\frac{1}{h_{i}}+\frac{e_{1}}{k_{1}}+\frac{e_{2}}{k_{2}}+\frac{1}{h_{e}}}
$$

Donde:

$\boldsymbol{h}_{\boldsymbol{n}}=$ Coeficiente de convección al interior y exterior $\left[\mathrm{W} / \mathrm{m}^{2} \mathrm{~K}\right]$.

$\boldsymbol{e}_{\boldsymbol{n}}=$ Espesor $[\mathrm{m}]$.

$k_{n}=$ Conductividad térmica $[\mathrm{W} / \mathrm{mK}]$.

En la Tabla 3, se pueden observar los parámetros tomados para determinar el coeficiente global de transferencia de calor.

\begin{tabular}{|l|r|l|}
\hline Parámetro & Valor & Unidades \\
\hline $\boldsymbol{h}_{\boldsymbol{i}}$ & 40 & $\mathrm{~W} / \mathrm{m}^{2} \mathrm{~K}$ \\
\hline $\boldsymbol{h}_{\boldsymbol{e}}$ & 6.15 & $\mathrm{~W} / \mathrm{m}^{2} \mathrm{~K}$ \\
\hline $\boldsymbol{e}_{\boldsymbol{1}}$ & 0.003 & $\mathrm{~m}$ \\
\hline $\boldsymbol{e}_{\boldsymbol{2}}$ & 0.02 & $\mathrm{~m}$ \\
\hline$k_{1}$ & 0.4 & $\mathrm{~W} / \mathrm{mK}$ \\
\hline$k_{2}$ & 0.038 & $\mathrm{~W} / \mathrm{mK}$ \\
\hline
\end{tabular}

Tabla 3 Parámetros para determinar U

Fuente: Elaboración Propia

\section{Carga térmica generada por cambios de aire}

La carga térmica generada por cambios de aire en el dispositivo se calculó mediante la siguiente ecuación:

$Q_{\text {cambios }}=v * f_{1} * f_{2}$

Donde:

$Q_{\text {cambios }}=$ Carga por cambios de Aire [W]. $v=$ Volumen $\left[\mathrm{m}^{3}\right]$.

$f_{1}=$ Factor por cambios de aire promedio en 24 hrs. para cuartos de almacenamiento arriba de 0 ${ }^{\circ} \mathrm{C}$ debido a la apertura de de puertas e infiltración. 
$f_{2}=$ Factor "Calor removido del aire de enfriamiento para cuartos de almacenamiento.

En la Tabla 4, se pueden observar los parámetros tomados para calcular la Carga Térmica generada por cambios de aire.

\begin{tabular}{|c|c|c|}
\hline Parámetro & Valor & Unidades \\
\hline$v$ & 0.003 & $\mathrm{~m}^{3}$ \\
\hline$f_{1}$ & 68 & Adimensional \\
\hline$f_{2}$ & 2.465 & $\mathrm{~W} / \mathrm{m}^{3}$ \\
\hline
\end{tabular}

Tabla 4 Parámetros para calcular la Carga Térmica generada por cambios de aire Fuente: Elaboración Propia

\section{Carga térmica total}

La carga térmica total se determinó mediante la suma de las cargas térmicas consideradas, esto es:

$$
Q_{\text {total }}=Q_{\text {producto }}+Q_{\text {paredes }}+Q_{\text {cambios }}
$$

\begin{tabular}{|l|r|l|}
\hline Parámetro & \multicolumn{1}{|c|}{ Valor } & Unidades \\
\hline$Q_{\text {producto }}$ & 0.1 & $\mathrm{~W}$ \\
\hline$Q_{\text {paredes }}$ & 4.7 & $\mathrm{~W}$ \\
\hline$Q_{\text {cambios }}$ & 5.56 & $\mathrm{~W}$ \\
\hline$Q_{\text {total }}$ & 10.7 & $\mathrm{~W}$ \\
\hline
\end{tabular}

Tabla 5 Carga Térmica Total Fuente: Elaboración Propia

\section{Elección de la celda Peltier}

Una vez calculada la carga total que el sistema debe retirar para mantener las temperaturas de operación, se seleccionó la celda Peltier a utilizar en el sistema. El sistema debe mantener temperaturas de entre $2^{\circ} \mathrm{C}$ y $8^{\circ} \mathrm{C}$; removiendo hasta $10.7 \mathrm{~W}$, a las máximas condiciones de operación. La temperatura exterior a la que se llevó el análisis es de $35^{\circ} \mathrm{C}$ considerando esta una condición extrema.

Para el presente trabajo, se estableció utilizar celdas Peltier estándar debido a su accesibilidad en el mercado y buena refrigeración. Estas celdas pueden ser alimentadas con $12 \mathrm{~V}$ para lo cual su uso energético es factible (Godoy,2016).

A continuación, en la Tabla 5 se muestran las especificaciones de una celda Peltier estándar TEC-1276:

\begin{tabular}{|l|l|l|}
\hline \multicolumn{2}{|c|}{ Especificaciones } & \multicolumn{1}{c|}{ Condiciones } \\
\hline I máx. & $6 \mathrm{~A}$ & $\mathrm{Th}=30{ }^{\circ} \mathrm{C}$ \\
\hline V máx. & $15 \mathrm{~V}$ & $\mathrm{Th}=30{ }^{\circ} \mathrm{C}$ \\
\hline$\Delta \mathrm{T}$ máx. & $\geq 67{ }^{\circ} \mathrm{C}$ & $\mathrm{Qc}=0, \mathrm{Th}=30{ }^{\circ} \mathrm{C}$ \\
\hline Q máx. & $51,4 \mathrm{~W}$ & $\Delta \mathrm{T}=0{ }^{\circ} \mathrm{C}, \mathrm{Th}=30{ }^{\circ} \mathrm{C}$ \\
\hline Tr & \multicolumn{2}{|c|}{ Desde -50 hasta $100{ }^{\circ} \mathrm{C}$} \\
\hline Dimensiones & $40 \times 40 \mathrm{~mm}$ \\
\hline
\end{tabular}

Tabla 6: Especificaciones de una celda Peltier TEC-1276 Fuente: Godoy, 2016

\section{Energía consumida por el dispositivo}

Con la gráfica universal de rendimiento (figura 3) y la Tabla 6 , se es posible conocer la intensidad de corriente a la que la celda Peltier debe operar para que remueva el calor del sistema y así poder calcular la Potencia Eléctrica que se requiere suministrar al contenedor de vacunas.

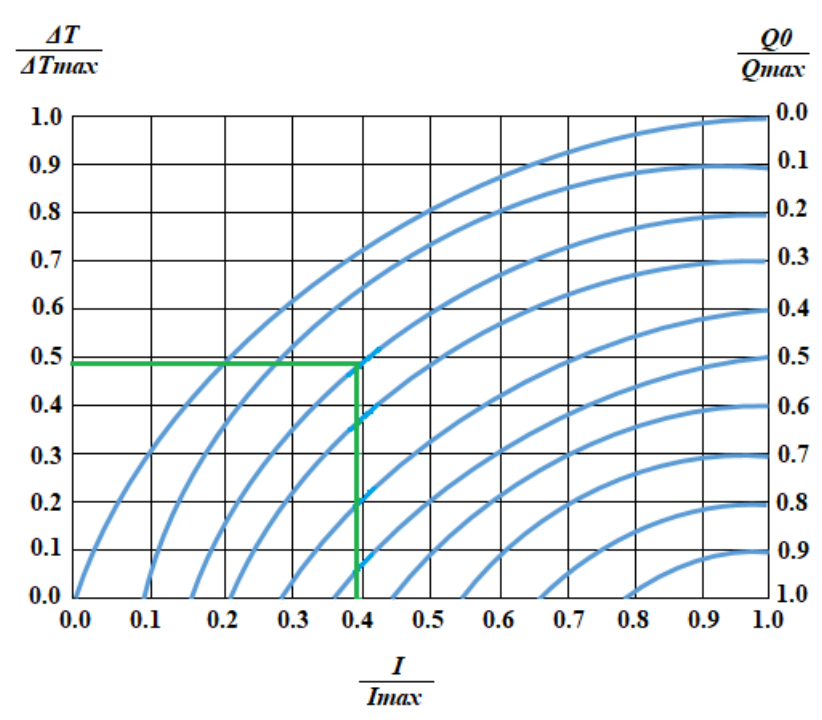

Figura 3 Curvas universales de rendimiento Fuente: Meerstetter Engineering, 2016

En la Tabla 7, se pueden observar los parámetros utilizados para encontrar el valor de la intensidad de corriente.

\begin{tabular}{|l|r|l|}
\hline Parámetro & Valor & Unidades \\
\hline$\Delta \boldsymbol{T}_{\max }$ & 67 & ${ }^{\circ} \mathrm{C}$ \\
\hline$\Delta \mathbf{T}$ & 33 & ${ }^{\circ} \mathrm{C}$ \\
\hline $\boldsymbol{Q}_{\max }$ & 51.4 & $\mathrm{~W}$ \\
\hline $\boldsymbol{Q}_{\mathbf{0}}$ & 10.7 & $\mathrm{~W}$ \\
\hline$I_{\max }$ & 6 & $\mathrm{~A}$ \\
\hline $\boldsymbol{I}$ & $\mathbf{2 . 4}$ & $\mathrm{A}$ \\
\hline
\end{tabular}

Tabla 7 Parámetros para encontrar el valor de la Intensidad de Corriente

Fuente: Elaboración Propia

La Potencia Eléctrica requerida por el sistema de refrigeración se calculó mediante la siguiente ecuación: 
$P=V * I$

Donde:

$P=$ Potencia eléctrica $[\mathrm{W}]$

$V=$ Voltaje máximo [V]

$I=$ Intensidad de corriente [A]

\section{Elección del panel fotovoltaico}

El panel fotovoltaico propuesto ha sido seleccionado bajo los siguientes parámetros: tipo de panel, eficiencia energética y potencia nominal de operación.

Se propuso un panel solar comercial liviano y flexible para una instalación rápida y fácil, con diseño plegable. En la tabla 8, se muestran las especificaciones del panel fotovoltaico.

\begin{tabular}{|l|r|l|}
\hline \multicolumn{1}{|c|}{ Parámetro } & \multicolumn{1}{c|}{ Ualor } & Unidades \\
\hline Potencia eléctrica & 80 & $\mathrm{~W}$ \\
\hline $\begin{array}{l}\text { Corriente de funcionamiento } \\
\text { optimo }\end{array}$ & 4.44 & $\mathrm{~A}$ \\
\hline Voltaje de operación optimo & 18.9 & $\mathrm{~V}$ \\
\hline Eficiencia & $25 \%$ & Adimensional \\
\hline Peso & 1.8 & $\mathrm{Kg}$ \\
\hline Largo & 0.65 & $\mathrm{~m}$ \\
\hline Ancho & 0.61 & $\mathrm{~m}$ \\
\hline Área & 0.4 & $\mathrm{~m}^{2}$ \\
\hline
\end{tabular}

Tabla 8 Ficha técnica del panel fotovoltaico Fuente: Elaboración Propia

Se realizó una comparativa entre el panel fotovoltaico de $80 \mathrm{~W}$ y uno de $60 \mathrm{~W}$ para observar cuál de los dos era el más adecuado a utilizar.

\section{Resultados}

Los resultados se presentan en los siguientes gráficos que muestran el comportamiento de los principales parámetros que afectan el desempeño del sistema de refrigeración, a lo largo del año y del día con la mayor carga térmica, que fundamentan la viabilidad energética del diseño del transportador de vacunas.

\section{Cargas térmicas}

En el Gráfico 3, se pudo observar la Carga Térmica de un día promedio a lo largo de los meses del año, en el cuál se observó que los meses con mayor carga térmica son junio, julio y agosto, debido a que el gradiente de temperatura en estos meses es mayor.

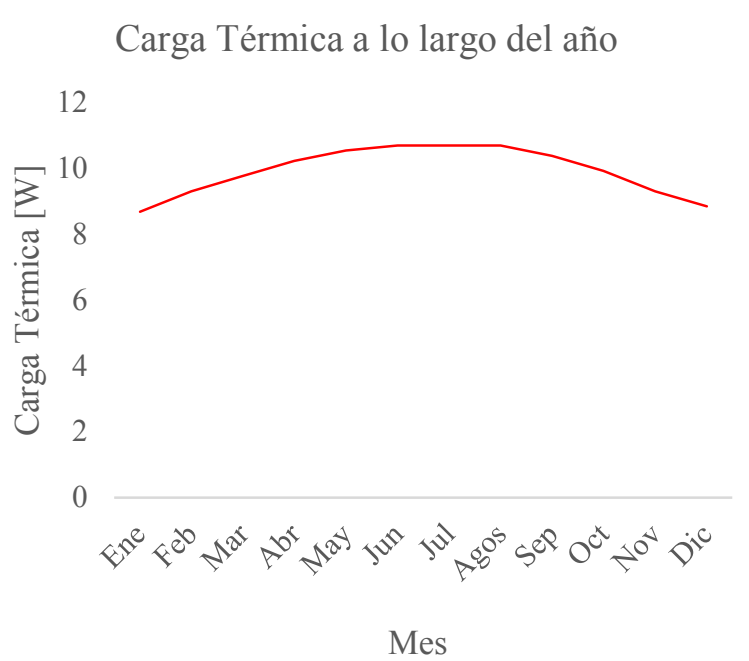

Gráfico 3 Carga térmica a extraer a lo largo del año Fuente: Elaboración Propia

En el Gráfico 4, se pudo observar la Carga Térmica horaria para un día de julio, que es el mes que presenta la mayor carga térmica. En esta gráfica se pudo observar que la mayor carga térmica a retirar es a las 16 y 18 horas.

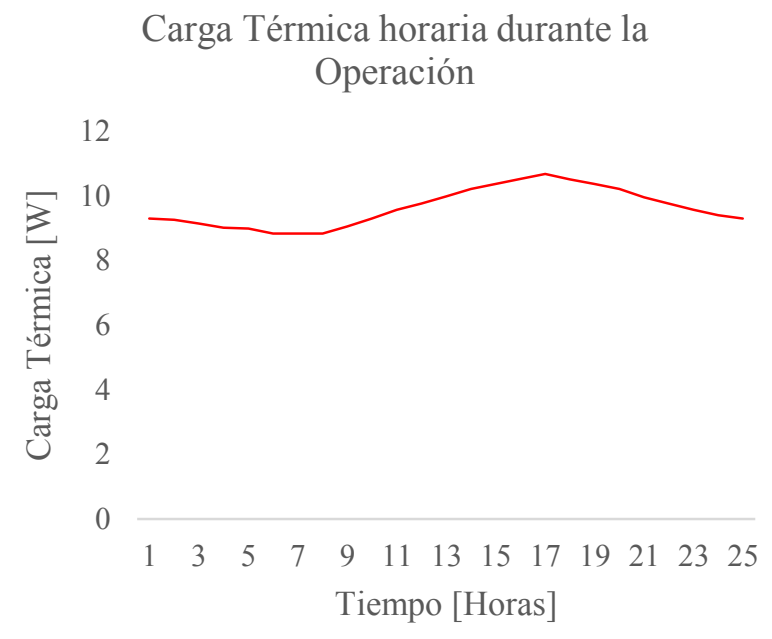

Gráfico 4 Carga Térmica horaria para un día de operación del mes de julio

Fuente: Elaboración Propia

\section{Energía Eléctrica requerida por el sistema de refrigeración}

En el Gráfico 5, se puede apreciar la energía eléctrica diaria que deberá ser suministrada al dispositivo por cada mes del año. En los días correspondientes a los meses de junio, julio y agosto existe una mayor demanda de energía eléctrica. 
Energía Eléctrica requerida por el sistema de Refrigeración a lo largo del año

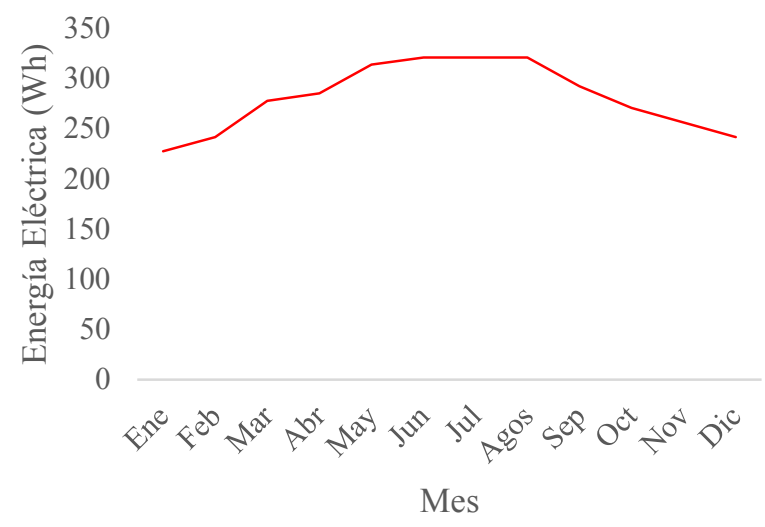

Gráfico 5 Energía eléctrica requerida a lo largo del año. Fuente: Elaboración Propia

En el Gráfico 6, se muestra la potencia eléctrica requerida por el sistema, durante las 8 horas de la jornada. Se pudo observar que la mayor potencia requerida es entre las 16 y 18 horas debido a las altas cargas térmicas.

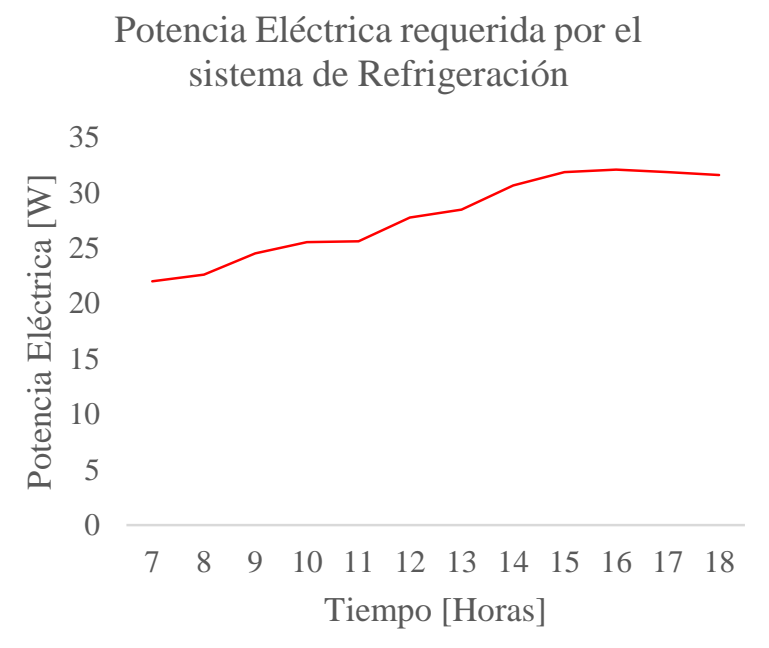

Gráfico 6 Potencia Eléctrica requerida de forma horaria a lo largo del día.

Fuente: Elaboración Propia

\section{Energía Eléctrica suministrada por el panel fotovoltaico}

En el gráfico 7 se muestra la energía eléctrica que proporcionará el panel fotovoltaico al dispositivo a lo largo de los meses del año, teniendo como mes máximo de aprovechamiento marzo.

\section{Energía Eléctrica suministrada por el panel fotovoltaico}

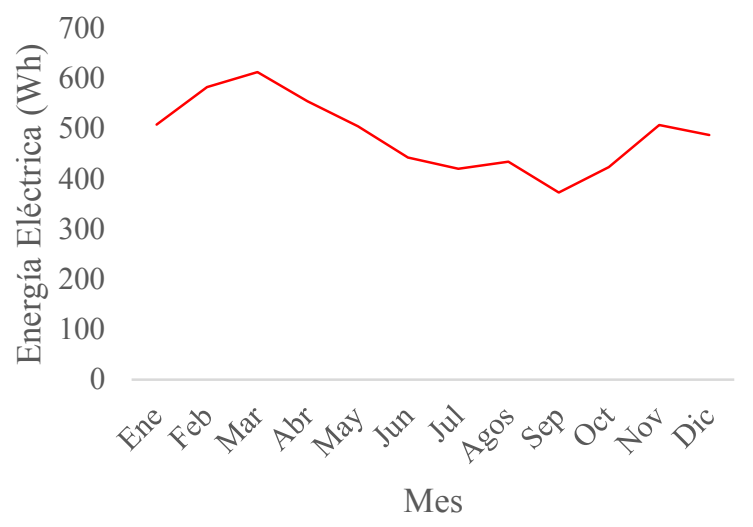

Gráfico 7 Energía Eléctrica suministrada a lo largo del año.

Fuente: Elaboración Propia

En el gráfico 8 , se puede observar la potencia eléctrica diaria generada por el panel fotovoltaico. Se pudo observar que entre las 13 y 14 horas se obtuvo la máxima potencia eléctrica proporcionada por el panel fotovoltaico.

\section{Potencia Eléctrica suministrada por el} panel

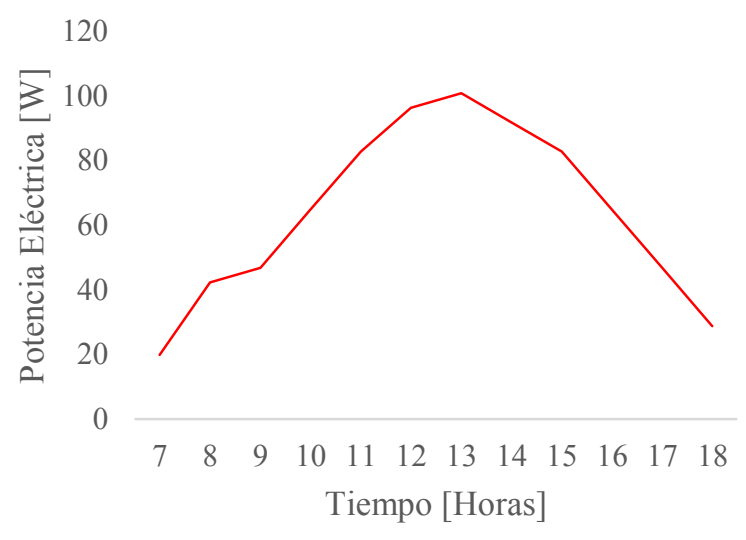

Gráfico 8 Potencia Eléctrica suministrada por el panel. Fuente: Elaboración Propia

\section{Evaluación Energética del Sistema}

En el gráfico 9, se muestra una comparativa entre la energía eléctrica requerida por el contenedor de vacunas a lo largo del año y la energía eléctrica suministrada con dos opciones de paneles fotovoltaicos; uno de $80 \mathrm{~W}$ y uno de 60 W.

Se pudo observar que la energía que suministra el panel de $80 \mathrm{~W}$, en todos los meses es mayor a la que el sistema requiere, en cambio con el panel de $60 \mathrm{~W}$ solo se suministra la energía eléctrica necesaria en los meses de enero, febrero, marzo, abril, noviembre $\mathrm{y}$ diciembre.

VALLE-HERNÁNDEZ, Julio, LOZANO-OLMEDO, Britania Junery, DÍAZ-MONROY, Joanna y ROMÁN-AGUILAR, Raúl. Diseño y análisis energético de un sistema de refrigeración autónomo para el traslado de vacunas. Revista de Aplicaciones de la Ingeniería. 2019. 


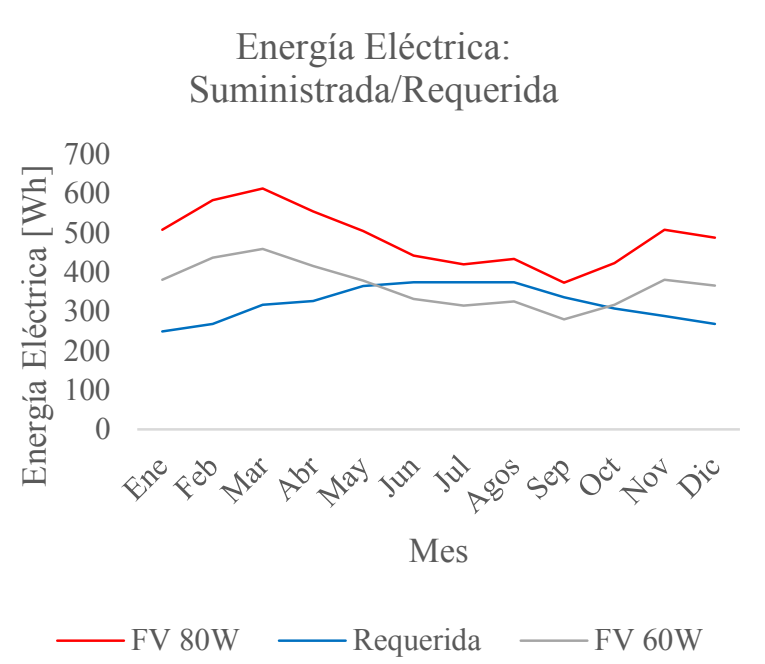

Gráfico 9 Gráfica comparativa de la Energía Eléctrica. Fuente: Elaboración Propia

En el gráfico 10 y 11, se muestran las comparativas entre la potencia eléctrica requerida por el contenedor y la potencia eléctrica suministrada por los paneles fotovoltaicos, para un día promedio de marzo y julio, respectivamente.

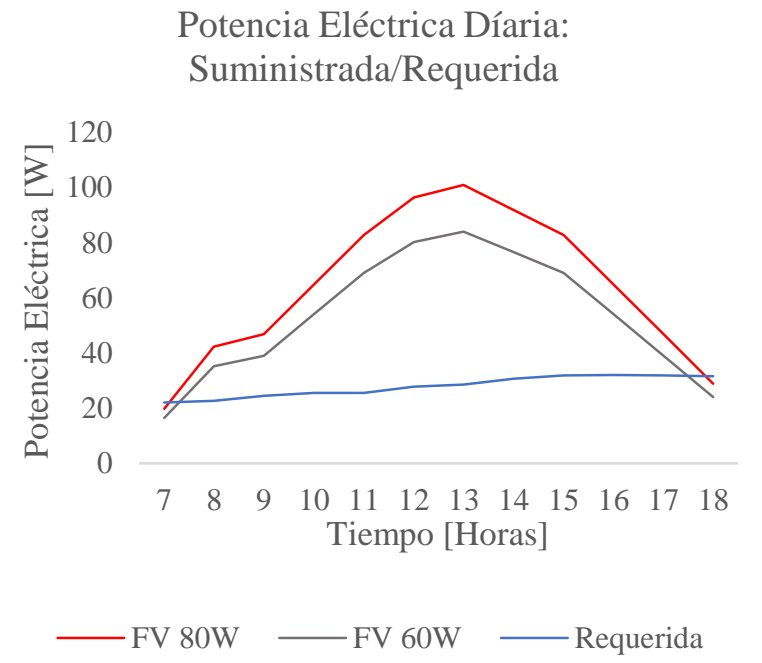

Gráfico 10 Gráfica comparativa de la Potencia Eléctrica para un día de marzo

Fuente: Elaboración Propia

En el gráfico 10 se puede observar que la potencia suministrada por cualquiera de los dos paneles satisface la demanda de energía del dispositivo, durante prácticamente todas las horas de la jornada de un día de marzo que es cuando se tiene la mayor irradiación solar del año. En tanto, en el gráfico 11 puede observarse que, durante el mes de julio, donde se tiene menor irradiación solar, sólo el panel fotovoltaico de $80 \mathrm{~W}$ sería una opción para cubrir la demanda de potencia eléctrica en un horario de 8:00 a 16:00.
El panel de $60 \mathrm{~W}$ solo cubriría la demanda de potencia de 10:00 a 15:00, por lo que requeriría de una batería de respaldo.

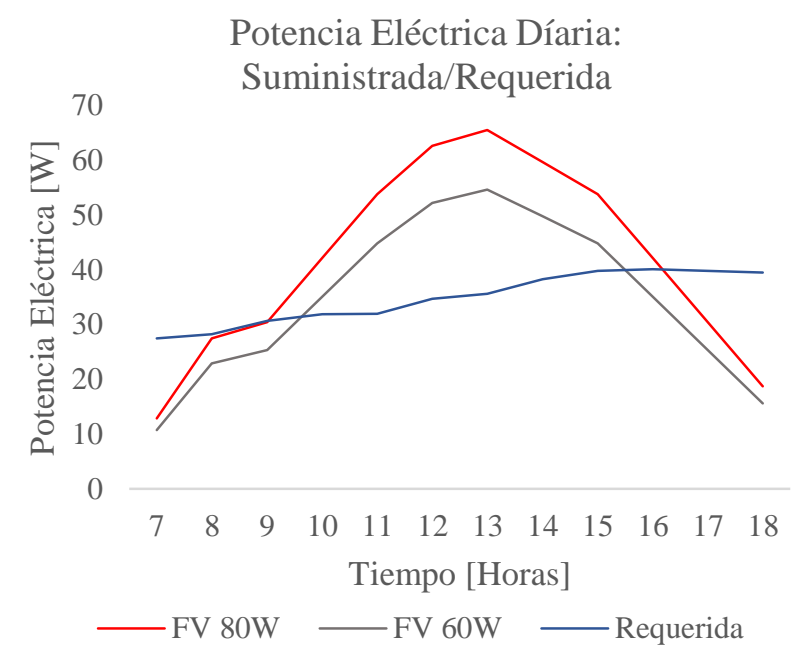

Gráfico 11 Gráfica comparativa de la potencia eléctrica para un día de julio

Fuente: Elaboración Propia

\section{Conclusiones}

En base a los resultados se puede afirmar que el diseño del sistema de refrigeración propuesto es óptimo desde el punto de vista energético. El diseño permite reducir las cargas térmicas generadas por transmisión de paredes y por infiltraciones de aire exterior. Lo anterior se consigue reduciendo al mínimo el área superficial, por sus dimensiones con una altura de $0.226 \mathrm{~m}$ y un radio de $0.065 \mathrm{~m}$ que otorgan la menor área de transferencia de calor para el volumen dado, así como por un sistema dispensador de vacunas que con dos niveles de 5 vacunas cada uno. Estos niveles se pueden girar para extraer una sola vacuna sin tener que abrir por completo el contenedor y generar infiltraciones del exterior.

Con este diseño, el sistema tiene la capacidad de refrigerar $100 \mathrm{ml}$ de vacunas que están contenidas en frascos de $5 \mathrm{ml}$ de capacidad, es decir, 20 frascos. El dispositivo opera expuesto a una temperatura máxima externa de $35^{\circ} \mathrm{C}$, reduciendo la carga térmica total a un máximo de $10.7 \mathrm{~W}$, que le permite mantener temperaturas internas de entre $2{ }^{\circ} \mathrm{C} \mathrm{y} 8{ }^{\circ} \mathrm{C}$. A si mismo de la evaluación energética tenemos que con un panel de $80 \mathrm{~W}$ se cubre por completo y de sobra la demanda de energía eléctrica para todas las condiciones de operación. Mientras que con un panel de $60 \mathrm{~W}$, que reduce peso del sistema y costos de inversión, se satisfacen casi todas las condiciones de operación, excepto para los meses de junio, julio y agosto que es cuando mayor carga térmica debe ser extraída.

VALLE-HERNÁNDEZ, Julio, LOZANO-OLMEDO, Britania Junery, DÍAZ-MONROY, Joanna y ROMÁN-AGUILAR, Raúl. Diseño y análisis energético de un sistema de refrigeración autónomo para el traslado de vacunas. Revista de Aplicaciones de la Ingeniería. 2019. 
Cabe mencionar que el sistema no requiere de baterías de almacenamiento durante la operación con un panel de $80 \mathrm{~W}$, pues satisface el requerimiento del sistema en tiempo real. Los resultados de este proyecto se pueden considerar un precedente para la implementación de sistemas móviles de almacenamiento refrigerados con energía solar, si bien los resultados son preliminares, son la base de un diseño y análisis energético viables que pueden ser comprobados en un futuro.

\section{Referencias}

ABB. (2009). Best-Practice Guide. Pharmaceutical-Chain Temperature Control and Recording. Cambridgeshire, UK. Recuperado de: https://library.e.abb.com/public/b0c7522c15 decafcc125766d0055530f/Best\%20Use\%20Gui de.pdf

BOHN. (2005). Manual de ingeniería. México. Recuperado de: www.bohn.com.mx

Diebel, J. (2019). Weather Spark. Minneapolis, Minnesota. Recuperado de: es.weatherspark.com/

Godoy Vaca, L. F. (2016). Diseño, construcción y evaluación energética de una cámara con celdas Peltier (efecto termoeléctrico) para refrigeración de vacunas. 130 hojas. Quito EPN.

HVACR. (2012). Carga térmica. México. Recuperado de: www.mundohvacr.com.mx/ 2012/06/carga-termica/

Incropera, F. (1999). Fundamentos de transferencia de calor. México, Prence Hall.

Meerstetter Engineering. (2016). TEC/Peltier Element Design Guide. Rubigen, Suiza. Recuperado de: www.meerstetter.ch/compen dium/tec-peltier-element-desing-guide

Nasa Power. (2019). Prediction Of Worldwide Energy Resource. Recuperado de: power.larc.nasa.gov/data-access-viewer/

OMS. (2019). Vacunas. Organización Mundial de la Salud. Recuperado de: www.who.int/topics/vaccines/es/

Solar fotovoltaica. (2002). Manuales sobre energía renovable: Solar Fotovoltaica. $1^{\circ}$ Edición, 6-7
S\&P. (s.f.). Hojas técnicas: Leyes de los ventiladores. Barcelona, España. Recuperado de: www.solerpalau.com/es-es/hojas-tecnicasleyes-de-los-ventiladores/

Villarrubia, M. (2001). La refrigeración termoeléctrica. Montajes e instalaciones: Revista técnica sobre la construcción e ingeniería de las instalaciones. 31(346), 65-72.

Winder, E., Ellis, A. \& Lisensky, G. (s.f.). Thermoelectric Devices: Solid-State Refrigerators and Electrical Generators in the Classroom. Wisconsin, EE.UU. Recuperado de: http://www.intalek.com/Index/Projects/Researc $\mathrm{h} /$ ThermoelectricDevices.PDF 